\title{
A comparative review of outcomes of triune grommets at 1-2 years post insertion.
}

\author{
Tammie MacFie ${ }^{1}$, Gemma Oades ${ }^{1}$, Frances Rowat ${ }^{1}$, Gaurav Kataria ${ }^{1}$, and Shadaba \\ Ahmed ${ }^{1}$ \\ ${ }^{1}$ Royal Lancaster Infirmary
}

June 13, 2020

\begin{abstract}
* NICE recommend that children with persistent bilateral otitis media with effusion (OME) documented over a 3-month period and associated hearing impairment should be considered for surgical intervention. ${ }^{*}$ Treatment of OME often requires repeated tubulation of the tympanic membrane if the grommets are extruded before the disease of the middle ear has remitted. $*$ Triune tubes appear to remain in situ and functional for longer than the standard ventilation tubes. * Use of triune grommets may reduce need for repeated tubulation of the tympanic membrane. * Complications from infection or recurrent effusion were similar, regardless of type of grommet used.
\end{abstract}

Key points

- NICE recommend that children with persistent bilateral otitis media with effusion (OME) documented over a 3-month period and associated hearing impairment should be considered for surgical intervention.

- Treatment of OME often requires repeated tubulation of the tympanic membrane if the grommets are extruded before the disease of the middle ear has remitted.

- Triune tubes appear to remain in situ and functional for longer than the standard ventilation tubes.

- Use of triune grommets may reduce need for repeated tubulation of the tympanic membrane.

- Complications from infection or recurrent effusion were similar, regardless of type of grommet used.

\section{Introduction}

Otitis media with effusion (OME) is a common early childhood condition where an accumulation of fluid within the middle ear space affects the hearing.

National Institute of Clinical Excellence (NICE) recommend that children with persistent bilateral OME documented over a period of 3 months, and with a hearing level in the better ear of 25-30 dBHL or worse should be considered for surgical intervention with the insertion of ventilation tubes, or grommets, recommended (1).

There are 3 main types of grommet used at UHMBT. These are Shepard (Figure 1), Shah (Figure 2) and, more recently, Triune tubes (Figure 3). Shepard and Shah grommets usually stay in place for 9-12 months before being extruded, after which they will be replaced if still required. The Triune ventilation tube was designed to address complications of the standard long-term T-Tubes, specifically perforations. Unlike the Shepard and Shah tubes, the Triune has three flanges to keep the tube in a perpendicular relationship to the eardrum, designed to prevent unnecessary erosion of the tympanic membrane. Triune tubes are thought to remain in situ for around 30 months before they extrude. Triune tubes are also thought to produce fewer perforations than other ventilation tubes whilst also allowing a longer period of ventilation (3)

The aim of this audit was to evaluate the longevity and efficacy of the Triune tube vs conventional grommets. 


\section{Methods}

\subsection{Ethical considerations}

This study took the form of an audit so National Research Ethics Service approval was not required.

\subsection{Data collection}

University Hospitals of Morecambe Bay NHS Foundation Trust (UHMBT) in North West England provides services in South Cumbria and North Lancashire in the Morecambe Bay area. Data were collected from patients attending the Royal Lancaster Infirmary.

This was a retrospective study of patients with triune tubes placed between October 2015 and August 2019. Comparison was made to 10 randomly selected control subjects, all of whom had Shah or Shepard grommets inserted during 2018 at UHMBT.

Indications for insertion of a ventilation tube were either glue ear, recurrent ear infections or tympanic membrane retraction.

Outcomes were based on whether the grommets remained in place and were patent at follow up checks 1-2 years post insertion. Note was also made of any complications the patient may have experienced.

3 Results

A total of thirty-one patients had triune tubes placed during October 2015 and August 2019, but only fourteen patients were included in the study as they had documented follow up between 12- 24 months.

Of the 10 control patients only five had documented follow up between 12- 24 months.

The mean follow-up time for all patients was 17 months.

The results show that $43 \%(6 / 14)$ of patients that had triune tubes inserted had at least one tube in situ and functional at follow up. Only $20 \%(1 / 5)$ of patients that had received the standard grommets had at least one tube in situ and functional at follow up.

$14 \%(2 / 14)$ of the patients with triune tubes had both tubes in situ at $>12$ months. None of the patients with control grommets had both tubes present at $>12$ months.

There were similar rates of infections in both groups. $20 \%(1 / 5)$ of the control group had documented infections, compared to $21 \%(3 / 14)$ of triunes group.

Recurrent effusion was documented in $20 \%$ (1/5) of the control group compared with $14 \%(2 / 14)$ of patients with triunes tubes.

The data that supports the findings of this study are available in the supplementary material of this article.

\section{Discussion}

\subsection{Synopsis of key findings}

Our study shows that there is a difference in outcome in patients receiving triune ventilation tubes compared to patients receiving Shah or Shepard ventilation tubes.

The key difference is that triune tubes appear to remain in situ and functional for longer than the standard ventilation tubes. Complications from infection or recurrent effusion were similar between the two groups.

\subsection{Comparisons with other studies}

Few research articles have documented the use of triune tubes in clinical practice. One describes them as a 'safe' option where myringotomy is indicated and finds the complications comparable to t-tubes and grommets (2). Hill (2006) published findings suggesting that triunes can provide ventilation for around 30 months. This study also showed a $3.0 \%$ perforation rate with triune tubes compared to $16.6 \%$ for standard 
long-term T-Tubes - the difference was attributed to the conformity of the triune tube to the convex tympanic membrane (3).

\subsection{Clinical applicability of the study}

Insertion of ventilation tubes to treat persistent OME is one of the commonest surgeries performed.

Our experience at UHMBT suggests that recurrent short-term grommet insertion with Shah or Shepard grommets carries a higher risk of residual perforation, implantational cholesteatoma and atelectasis of the tympanic membrane. There are also the associated risks with repeated general anaesthetic. The alternatives to Shah or Shepard would be T-tube but these also carry the above risks as well as having to be surgically removed with the possibility of a myringoplasty to close the residual perforation.

As triune tubes remain in situ for up to 30 months before extrudation these risks are reduced and therefore more likely that re-accumulation of OME does not occur and therefore normal eustachian tube function returns.

From a practical point the Triune tube is easier to insert, does not require additional equipment or prolonged anaesthetic time. It is suitable for children with narrow canals and no allergic reactions or extrusions secondary to rejection have been noted for the Triune. Like standard grommets it can be placed into the inferior quadrants, even in atelectic tympanic membranes without appearing to have a detrimental effect on hearing. Compared to standard grommets, which are often difficult to visualise, the Triune's colour is highly visible making it easier to assess its presence and position. The lumen size of the Triune also makes it suitable to administer topical antibiotics. Triunes can also be placed in revision cases with similar outcomes to primary cases.

Our pilot study has shown that triune tubes remain in situ and functional for longer than standard ventilation tubes. Careful consideration should be given to the type of ventilation tube used to prevent the need for repeated intervention.

\subsection{Limitations}

This study has its limitations. The most obvious one is the sample size. This is largely as a result of operator preference for selection of type ventilation tube and patients being lost to follow-up.

\section{Conclusions}

To our knowledge, our pilot study is the first in the UK to compare the longevity and efficacy of the Triune tube versus conventional grommets. This study found no evidence for difference in rates of infection or recurrent effusion between the triune group and control group, but did find that the triune grommets remained in situ for longer, and may therefore be beneficial to avoid repeated tubulation of the tympanic membrane.

\section{References}

1. NICE. 2008. Otitis media with effusion in under 12s: surgery. NICE Clinical guideline [CG60]).

2. Schramm, J.C., Person, A. Burt, A.J. \& Yonkers, A.J. Triune tube outcomes. 2014. Otolaryngol Head Neck Surg. 2014. 151(1):49.

3. Hill, F. The Triune, a new silicone tympanostomy tube. Otolaryngol Head Neck Surg. 2006 Mar;134(3):524-5.

\section{Hosted file}

Triune Figures.docx available at https://authorea.com/users/332593/articles/459022-acomparative-review-of-outcomes-of-triune-grommets-at-1-2-years-post-insertion 